\title{
Reward prediction error and declarative memory
}

5

6

7

8

Kate Ergo ${ }^{1}$, Esther De Loof ${ }^{1} \&$ Tom Verguts ${ }^{1}$

9

10

$11{ }^{1}$ Department of Experimental Psychology, Ghent University, Henri Dunantlaan 2, B-9000

12 Ghent, Belgium

13

14

15

16

17

18

19 Correspondence: tom.verguts@ugent.be

20 Tom Verguts

21 Department of Experimental Psychology

22 Ghent University

23 Henri Dunantlaan 2, B-9000 Ghent, Belgium

24 Phone number: +3292646408 
Reward prediction error and declarative memory

Abstract

26 Learning based on reward prediction error (RPE) was originally proposed in the context of

27 non-declarative memory. We postulate that RPE may support declarative memory as well.

28 Indeed, recent years have witnessed a number of independent empirical studies reporting

29 effects of RPE on declarative memory. In this paper, we provide a brief overview of these

30 studies, point out emerging patterns, and identify open issues such as the role of signed

31 versus unsigned RPEs in declarative learning.

32

33 Keywords: reward prediction error, declarative memory, reinforcement learning 
A tennis player knows how to perform a perfect serve, and also knows the opponent's

name. But how are these two types of "knowing" similar, if at all? It is thought that the

human brain houses at least two broad and distinct memory systems [1], each with its own

learning algorithms and neural correlates. The first is non-declarative (or habit, or implicit)

memory (Glossary). The second is declarative (or, in humans, propositional, or explicit)

memory. The computational principle of reward prediction error (RPE)-based learning

$[2,3]$ is generally thought to drive non-declarative learning. We review recent evidence that

42

RPEs also drive declarative memory.

One of the most influential theories in current cognitive neuroscience is predictive coding $[4,5]$. According to this account, the brain generates predictions about its own percepts, actions, and cognition, in order to learn about, build models of, and navigate the world [6]. A key concept in predictive coding is the prediction error (PE). Specifically, in order to generate accurate predictions, the brain needs to set a number of parameters (e.g., encoded in its synaptic connections). PEs allow updating such parameters.

Predictions can be made about several variables, such as tomorrow's weather, the next action I (or somebody else) will perform, our partner's mood, and so on. One particularly relevant variable to make predictions about, is reward; a PE in reward (by definition) is a RPE. The concept of RPEs has been very influential in non-declarative learning. In particular, RPEs were implemented in a wide range of computational models. For example, to account for blocking in non-declarative learning, Rescorla and Wagner (RW; [7]; Box 1) developed their now-classic model according to which learning depends on PE. Specifically, synaptic strength increases when a reward is better than expected, but synaptic strength decreases when the reward is worse than expected. Hence, the valence of the RPE matters (signed RPE) (SRPE). Further computational development of RW led to the temporal 
difference (TD; Box 1) Reinforcement Learning model [3]. The TD model improved upon the RW model because it allows learning also when the reward is not immediately present. However, the main success of the RPE concept as implemented in TD was probably of an empirical nature. In particular, dopaminergic neurons in the ventral tegmental area (VTA) implement a TD-like RPE signature of reward processing [8,9]. In recent years, the role of TD-based RPEs in non-declarative learning has become well established in psychology, neuroscience, and Artificial Intelligence. For example, deep Reinforcement Learning models use TD-based RPEs to solve tasks (e.g., playing Atari games) that were long considered beyond the capacity of artificial agents $[10,11]$. In contrast to the RW and TD models that are SRPE based, Pearce and Hall proposed that learning occurs whenever reward is surprising (either better or worse, that is, different than expected; consistent with an unsigned RPE; Box 1) (URPE) [12]. It is noteworthy that normative, Bayesian models of learning exhibit features of both. For example, the Kalman filter [13] updates its estimates based on SRPEs, but its learning rate (i.e., the extent to which parameters (such as synaptic weights) are updated) is driven by uncertainty, which can be estimated via URPEs [14-16]. Empirical signatures of both SRPE and URPE have been observed in the brain [17].

\section{Reward Prediction Error in Declarative Learning}

Although the role of RPEs in non-declarative learning has been studied extensively and formalized in a number of computational models, their role in declarative learning has only recently become a topic of interest. Two main approaches exist for elucidating the RPE effect on declarative learning (for an overview, see Table 1). First, in the reward-prediction approach (Box 2), a statistical distribution determines the probability of reward. The participant knows or estimates this reward distribution. Thus, the participant can make a prediction about reward; and based on the prediction, a RPE can be generated. Studies using 
84

85

86

87

88

89

90

91

92

93

94

95

96

97

98

99

100

101

102

103

104

105

106

107

reward prediction can be approximately ordered based on the difficulty of this prediction, and we will discuss them in that order (easy to difficult). The study of [18] was one of the first to use such an approach. In an incidental memory task, each of three cues were linked to different reward values. A medium reward led to improved recognition when it was better than predicted (i.e., when it was preceded by a cue predicting low or medium reward) relative to when it was worse than predicted (i.e., preceded by a cue predicting high or medium reward), consistent with a SRPE effect. However, later work could not replicate the SRPE effect in this specific experimental paradigm $[19,20]$.

A second implementation of the reward-prediction approach is the recent variablechoice paradigm (Figure 1a (Key Figure) and Box 2; [21]). Here, participants learn DutchSwahili word associations under different RPE value conditions. See Figure 1a for an overview of all RPEs in this design. Predicting the reward probability is again quite easy; participants can deduce it from the number of eligible options. Behaviourally, memory performance showed a SRPE effect in declarative learning: Recognition accuracy and certainty increased linearly with larger and more positive RPEs (Figure 1b). These results were replicated with image-word associations [21] and face-word associations [22].

In another instantiation of the reward-prediction approach, participants actively track and estimate the reward probability distribution. Here, on each trial, they experience a RPE relative to that (estimated) distribution (Figure 1c, Figure 1d and Box 2) [23-25]. Based on this feedback, they can update their estimate for subsequent trial estimates. For example, in [23], participants estimated the (fixed) probability of reward attached to specific stimuli. At reward feedback, a trial-novel image was presented. Subsequent memory performance for these trial-novel images displayed a SRPE effect, which was more pronounced in adolescents than in adults. 
In [25], participants tracked the reward associated with different indoor and outdoor scenes. A clear URPE effect was observed: Scenes associated with a higher URPE during the initial task (i.e., with more surprising rewards, in either positive or negative direction), were afterwards better remembered (Figure 1c-d).

[26] used a reward-prediction paradigm to disentangle effects of SRPE, surprise (which corresponds to URPE), and uncertainty. Unlike in the other paradigms just discussed, reward probability was not fixed, but instead jumped to a different level at unpredictable time points in the experiment. Only SRPE had an effect on subsequent memory (Figure 1f) (see also [27]). Finally, in [24] the reward probability would fluctuate slowly but unpredictably on each trial, making the reward-prediction task very challenging. In this experiment, unlike the other discussed paradigms, a negative effect of (S)RPE was observed. Specifically, trials (and participants) with stronger and more positive RPEs, were associated with impaired declarative learning.

As a second approach, in a multiple-repetition paradigm (Box 2), a set of general information questions are repeated a number of times. Trial-specific confidence ratings ("How certain are you that you answered correctly?") and feedback are used to compute trialspecific PEs. Given that being correct is rewarding [28], these PEs can be considered as RPEs. The researchers use these RPEs to predict accuracy on subsequent presentations of the same general information questions. Here, a URPE effect is typically observed. In particular, the hypercorrection effect obtained in this multiple-repetition paradigm entails that errors made with high confidence are beneficial for memory [29-33]. High-confidence errors are exactly those trials during which positive feedback was expected but not obtained; thus, this effect is consistent with a URPE effect. Also [34] observed a hypercorrection effect and interpreted it as a URPE. Additionally, in their experiment, participants received false feedback on a small fraction of trials (i.e., trials that were answered correctly but labeled as 
133 false), and received novel feedback (i.e., a novel "correct" answer) on those trials. In those

134 false-feedback trials, a URPE effect was also observed: On trials that were answered with

135 high certainty but that were not rewarded (high URPE), the novel feedback was subsequently

136 recalled more confidently.

137 Overviewing and categorizing these paradigms, we note that a main difference

138 between the reward-prediction and multiple-repetition approaches is the origin of the RPE:

139 An independent reward generation mechanism in the former, and the participant's own

140 confidence in his or her memory in the latter. Another difference is that, in the reward-

141 prediction approach, RPEs are usually computed or estimated, whereas RPEs are deduced

142 from confidence measures in the multiple-repetition approach. There are some exceptions to

143 the latter rule: For example, [25] implemented a reward-prediction paradigm where

144 confidence is used to calculate a RPE. Finally, in the reward-prediction paradigm,

145 memoranda are usually trial-unique, whereas (by definition) they are not in the multiple-

146 repetition approach. These are just a few of the relevant dimensions; we discuss some other

147 potentially relevant dimensions in the next section.

Open Issues

Despite the recent interest and steeply growing data set on RPEs that drive declarative memory, many uncertainties remain. We discuss a few of them in the next paragraphs.

\section{RPE: Signed or Unsigned?}

Studies with a multiple-repetition paradigm typically observed URPE (i.e., surprise)

157 Why do different designs generate SRPE versus URPE effects on declarative learning? One 
158 potentially relevant factor is the range of the RPEs probed. In particular, studies that found a

159 behavioral SRPE effect (i.e., most reward-prediction paradigms) might simply not have

160 investigated the full range of RPEs. In the variable-choice paradigm [21,35], this could be

161 tested by including a few non-rewarded one-option (high-certainty) trials. These highly

162 infrequent events would be accompanied by large negative RPEs.

163 However, this is unlikely to be the full story, because both RPE signatures have been

164 observed even within a single study. In an EEG study with the variable-choice paradigm [35],

165 an URPE pattern was observed during reward feedback in the theta (4-8 Hz) frequency band,

166 consistent with literature implying theta in URPE processing [36]. Instead, SRPE signatures

167 were found in the high-beta $(20-30 \mathrm{~Hz})$ and high-alpha $(10-15 \mathrm{~Hz})$ frequency ranges,

168 consistent with a functional role of both beta and alpha power in reward feedback processing

$169[37,38]$. Furthermore, in an fMRI study using a multiple-repetition paradigm, [34] found

170 SRPE-consistent activation in several areas (including striatum), but URPE signatures in

171 others (including insula). Together, these findings suggest that both SRPE and URPE are

172 important for declarative learning; and that we need an account identifying the functional role

173 of each, in time, (neural) space, and frequency band. The Bayesian learning model mentioned

174 in the introduction, which naturally incorporates both, may be a useful starting point in this

175 respect.

176

177 Timing Issues of RPEs

178 In most paradigms, a novel declarative memorandum is presented on each trial,

179 followed by a RPE, followed by declarative feedback about what the correct answer should

180 have been (see Figure 1a, word pair encoding for an example). Here, RPE can have either a

181 retrograde effect (if it interacts with the originally presented memoranda), or instead an

182 anterograde effect (if it interacts with the declarative feedback). Concerning the anterograde 
183 effect, in the variable-choice paradigm, the declarative feedback appeared either

184 simultaneously with the RPE (delay of $0 \mathrm{~ms}$; [21]), or with a delay of $3000 \mathrm{~ms}$ [35]. The fact

185 that we find very similar results in the two cases suggests that the timing of the RPE-

186 feedback interval is not very crucial, at least within the first few 100s of ms. An interesting

187 parallel can be drawn here with the test-potentiated learning effect from the declarative memory literature. Here, taking a test potentiates the learning of (old or novel) material that is subsequently presented [39,40]. Also for a retrograde effect (of RPE on originally presented memorandum), an interesting analogy can be made with earlier literature. In particular, [41]

found a retrograde effect of reward on declarative memory, with objects that were (temporarily) closer to (subsequent) reward being better remembered afterwards. In the reward-prediction approach, it remains to be shown which of these two (anterograde or retrograde effect of RPE) is crucial for driving the RPE-based declarative memory improvement.

A RPE can also appear at cue rather than at feedback. The only paper thus far investigating both cue- and feedback-locked RPE effects is [26]. These authors observed cuebut not feedback-locked RPE effects; however, in their experiment, there was both a cue- and a feedback-locked RPE on each trial. It is very well possible that an initial RPE suppresses a second RPE occurring (e.g., a few $100 \mathrm{~ms}$ later) in that same trial. We conclude that RPE timing issues need to be studied more systematically. In particular, if this research is to have 202 practical application in education, such studies will be imperative.

\section{RPE: Why and How?}

In non-declarative learning, a normative argument for why to use RPE is well established: Calculating RPE is necessary for online (i.e., while interacting with the world)

207 reward maximization [3]; this idea is inherent in the RW, TD, and Pearce-Hall models (Box 
208 1). Does this argument apply to declarative memory as well? An intuitive argument is that it makes sense to only remember stimuli (or more generally, episodes) that are associated with a reward level that is sufficiently different from what is already expected. Indeed, if a stimulus from some category is accompanied by reward each time it is encountered, it makes

212 little sense to explicitly remember each novel stimulus instance as a separate event once it has 213 already been learned.

Another issue is how RPE improves memory. One potential mechanism is via phaselocking to neural oscillations in specific frequency bands. In particular, neural theta phase synchronization may provide one (but not exclusive) solution: Brain areas in theta phase synchrony are thought to communicate and learn more efficiently [42], thus facilitating memory integration [43]. Indeed, episodic memory is enhanced when multimodal (audiovisual) stimuli are synchronously presented in theta phase; with stronger theta phase synchronization predicting better memory performance $[44,45]$. Dopaminergic midbrain neurons have also been found to phase-lock to (cortical) theta during encoding, with stronger 222 phase-locking during subsequently remembered (versus forgotten) memoranda [46]. Thus, it is possible that RPEs (via neuromodulatory signaling) increase theta synchrony, which subsequently allows the relevant brain areas to "glue" the episode together more efficiently [47]. The EEG variable-choice paradigm study mentioned above [35] provides preliminary evidence for this view. Further, computational models that consider RPE-theta interactions to drive learning, have started to appear [48].

Whereas dopaminergic RPEs likely support non-declarative learning via basal ganglia pathways, dopaminergic RPEs may support declarative memory via hippocampus [49]. Standard theory holds that (dopaminergic) VTA calculates SRPE, but a substantial number of URPE neurons have also been observed in VTA and nearby midbrain areas [50]. Moreover, also noradrenergic locus coeruleus projects to hippocampus and may thus exert URPE effects 
233 [51]. Earlier authors proposed that VTA-hippocampus interactions originate in hippocampus

234 [52]. We propose that VTA-hippocampus interactions may also originate in VTA, and that

235 SRPEs (encoded by VTA, possibly based on input from ventral striatum; [53]) and URPEs

236 (encoded in VTA and locus coeruleus) may modulate hippocampus for episodic memory

237 encoding. Consistently, a number of studies have demonstrated that midbrain VTA activation

238 (triggered by reward or by RPE) is associated with improved episodic learning [22,54,55].

\section{The Effect of Test Delay on Declarative Memory}

In declarative memory studies, participants are typically subjected to an implicit or

explicit memory test; either on the same day or after a considerable delay (ranging from a

243 few hours to a few weeks). If, as suggested above, SRPEs are encoded by dopamine neurons, then effects should be stronger with longer delays. Indeed, although early and late long-term memory effects both rely on dopamine, late effects have a stronger dependency on dopamine [49]. Consistently, an effect of reward in declarative learning is typically stronger after a delay [56,57]. However, a systematic comparison of the delay-by-RPE interaction on declarative memory remains to be carried out.

\section{Reconsolidation}

When information is retrieved from memory, it enters a plastic, labile state, allowing

252 the information to be changed, strengthened or weakened, a process called reconsolidation

253 [58,59]. This finding is most intensively studied in non-declarative memory [60], but is observed in declarative memory as well [61]. PE is required for reconsolidation [62] both in non-declarative [63] and in declarative memory [61,64]. Given the important role of RPE in declarative learning, and given that similar principles drive learning and reconsolidation [64],

257 we predict that RPE may modulate reconsolidation too. The multiple-repetition approach, 
where declarative memory is probed iteratively, can be considered as a first attempt at investigating RPEs in the context of reconsolidation. This remains, however, to be further investigated.

\section{Concluding Remarks and Future Perspectives}

Learning, RPEs, and declarative memory are sometimes treated as separate topics, each with their own prominent paradigms, findings, and theories. The current perspective suggests instead that they are intimately related. Briefly, learning is modulated by RPEs, and leads to (declarative) memory traces in the brain. We discussed a few recent paradigms that started to explore such interactions. In the Open Issues section, we highlighted a number of dimensions of those paradigms, that if addressed, could greatly facilitate further development of the research field. Although much remains to be found out, concrete models and predictions are beginning to emerge, with relevance for both Natural and Artificial Intelligence. We are excited about what the (near) future will bring in that respect, not only because of its conceptual unification, but also because of its promise for informing educational policy and practice.

\section{Glossary}

Declarative memory: Memory for facts and events ("knowing what"), that can (at least in humans) be (consciously) declared; it is typically considered to consist of episodic memory (memory for single episodes) and semantic memory (memory for information aggregated across several episodes). The process of acquisition of declarative memory is called declarative learning. Encoding declarative memories can happen rapidly, typically after only a single exposure (for both episodes and semantic content), and relies heavily on the hippocampus [65]. 
283 Non-declarative memory: Non-declarative learning is an umbrella term for the acquisition

284

285

of different types of knowledge, including procedural memory ("knowing how"). This involves acquiring a motor or cognitive skill (procedure) by means of repeated practice (e.g., learning to play tennis).

Prediction error: Difference between the actual value of some variable and predicted value of that variable (i.e., actual value minus predicted value).

Reward prediction error: Prediction error where the relevant variable is reward (i.e., actual reward - predicted reward). See also Prediction error.

Signed: In mathematics, signed means that the sign of a number is taken into consideration (e.g., $-3,+3$ ). In the context of SRPEs it indicates that we take the valence (positive versus negative RPEs) into account.

Theta phase synchronization: Synchronization of two brain areas in the theta frequency (4$8 \mathrm{~Hz}$ ). Such synchronization can be achieved by making the theta phase of the two areas identical, so that theta waves in both areas "go up and down" together.

Unsigned: Unsigned means that the sign is not considered (i.e., absolute value is taken, e.g., 3 and +3 both have an unsigned value of 3 ). See also Signed.

\section{Box 1. Models of Learning}

The Rescorla-Wagner model [7] describes learning the value (expected reward) of specific events (say, events A and B). This information is encoded in their associative strength to a "value" unit, symbolized as $w_{\mathrm{A}}$ and $w_{\mathrm{B}}$ for events A and B, respectively. Specifically, based on whether events $\mathrm{A}$ and $\mathrm{B}$ occur $\left(x_{\mathrm{A}}=1\right.$ and $x_{\mathrm{B}}=1$, respectively) or not $\left(x_{\mathrm{A}}=0\right.$ and $x_{\mathrm{B}}=0$, respectively), an additive prediction is made about the occurrence of reward $\left(V=x_{\mathrm{A}} \times w_{\mathrm{A}}+\right.$ $\left.x_{\mathrm{B}} \times w_{\mathrm{B}}\right)$. When reward finally occurs (or not), a reward prediction error is calculated $(R-V)$, where occurrence of reward (denoted $R$ ) is typically coded as $R=0$ (when there is no reward) 
308

309

310

311

312

313

314

315

or $R=1$ (when there is reward). This reward prediction error is then used to change the connection strength between cells encoding A and B on the one hand, and reward on the other: $\Delta w_{i}=\alpha \times x_{i} \times(R-V)$, with $i \in\{\mathrm{A}, \mathrm{B}\}$. After repeated application of this learning rule, the weights $w_{\mathrm{A}}$ and $w_{\mathrm{B}}$ allow the model to accurately predict reward, based on the (A, B) input combination.

Temporal Difference model [3]. The Rescorla-Wagner model can only learn from external feedback $(R-V)$. This is computationally inefficient because reward may be not delivered at each time point where relevant information is provided to the organism. In temporal difference learning, learning can also occur if the prediction of reward changes between two time points $t$ and $t+1$. Formally, the learning rule becomes (now with explicit time index $t$ ): $\Delta w_{i}(t)=\alpha \times x_{\mathrm{i}}(t) \times(R(t)+\gamma V(t+1)-V(t))$, with $i \in\{\mathrm{A}, \mathrm{B}\}$. If $\gamma=0$, the rule reduces to the Rescorla-Wagner rule. In case $\gamma>0$, learning can also proceed at times $t$ where no actual reward was delivered, rendering the algorithm more powerful than the Rescorla-Wagner rule.

Pearce-Hall model. According to this model [12], learning only occurs when a reward is surprising. Specifically, it uses the absolute value of a RPE (“different than expected” signal), consistent with an unsigned RPE approach. Formally, (one variant of) the learning rule can be written as: $\Delta w_{i}(t)=x_{\mathrm{i}}(t) \times R(t) \times|R(t)-V(t)|$.

\section{Box 2. How to Generate and Measure RPEs: Experimental Approaches}

Reward-prediction approach: Here, participants must both learn declarative information

(e.g., word pairs) and simultaneously estimate a (potentially non-stationary) reward distribution throughout the task [24-26]. In some cases, the correct RPE can be easily derived analytically; in other cases, RPE can only be calculated after fitting a reinforcement learning 
333 model, and deriving the RPEs from the model estimates [24,26]. One example of a reward-

334 prediction approach is the variable-choice paradigm. In the variable-choice paradigm [21,35]

335 (Figure 1a), participants learn stimulus pairs, such as Dutch-Swahili word pairs or image -

336 Swahili stimulus pairs [21]. In the former example, on each trial, a Dutch word is shown

337 together with four Swahili words. Critically, the number of eligible options is manipulated. In

338 the one-option, two-option, and four-option conditions, one, two, or four Swahili words are

339 eligible (framed), respectively; and the probability of choosing the correct translation is thus

$340100 \%, 50 \%$, or $25 \%$, respectively. Feedback is given on every trial. Signed and unsigned

341 trial-by-trial RPEs are calculated based on the difference between actual and predicted

342 reward (see Glossary). Memory is probed in a subsequent recognition test.

344 Multiple-repetition approach: Here, general information questions are repeatedly presented,

345 and a RPE is estimated based on previous presentations of each question. For example, in

346 [34], participants first studied a text, and subsequently received (multiple-choice) questions

347 about the text. After each question, they rated confidence and received feedback. The trial-

348 by-trial PE was calculated using the confidence rating and feedback. Hypercorrection effect 349 studies also typically use a multiple-repetition paradigm $[31,66]$. 
Reward prediction error and declarative memory
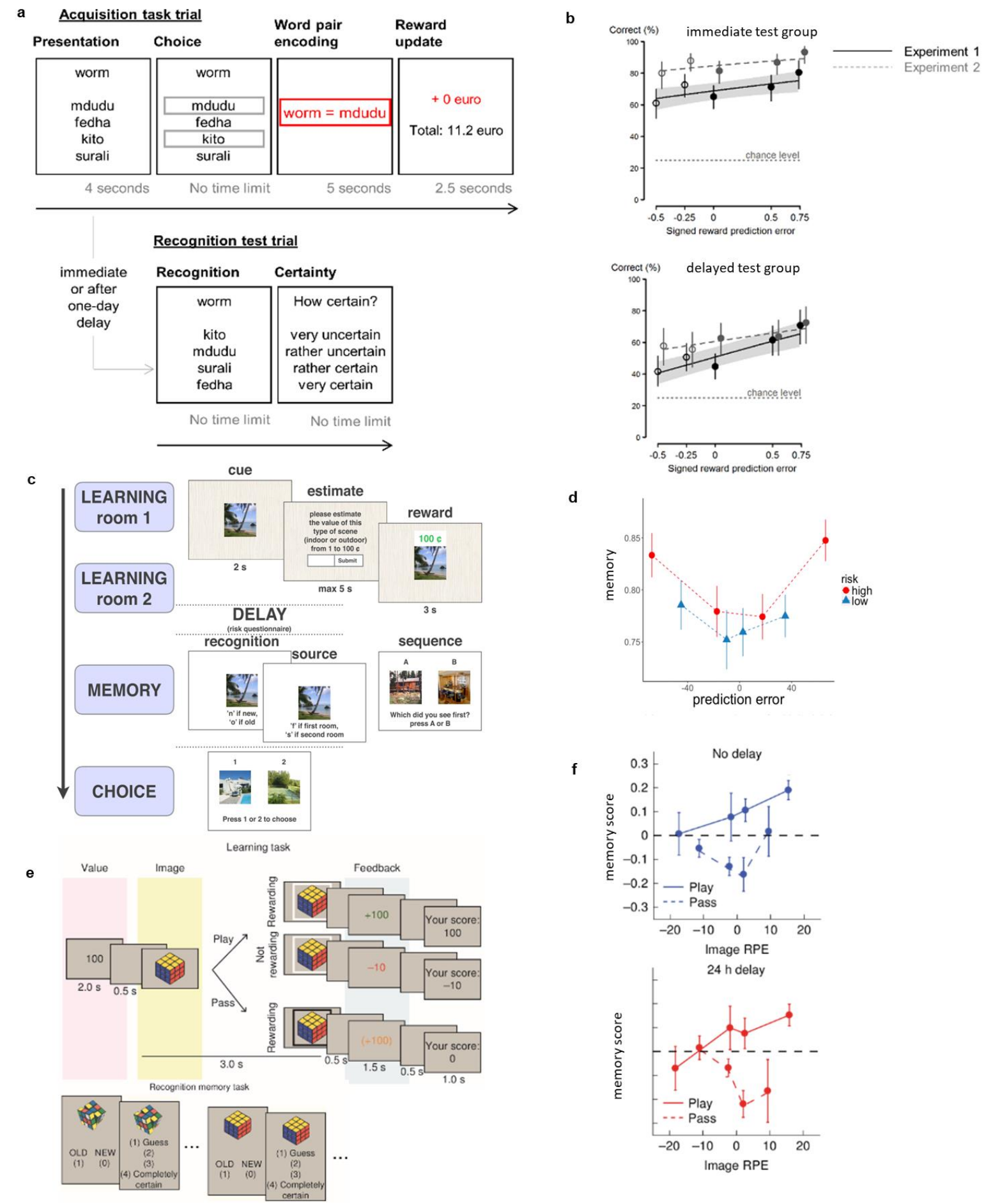

d
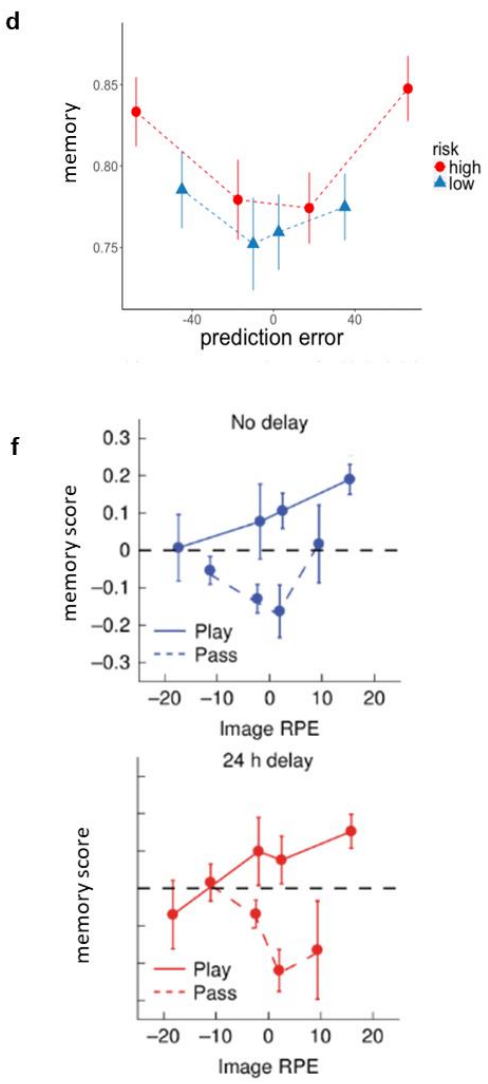

352 Figure Captions

353 Figure 1. RPE in declarative memory: Reward-prediction approach applied in three

354 paradigms and typical findings. a) Variable-choice paradigm and design from [21]. b)

355 Variable-choice paradigm behavioural results show a SRPE signature for recognition in both

356 the immediate and delayed test group; recognition of word pairs increased linearly with larger 
357 and more positive RPEs. c) Paradigm reproduced from [25]. d) [25] found a URPE (U-

358 shaped) signature; with memory improving for both large negative and large positive RPEs.

359 e) Paradigm reproduced from [26]. f) Jang et al. (2019) found a SRPE signature: Memory 360 score increased with increasing RPE.

361 
Table 1. Non-exhaustive overview of studies on RPE in declarative memory.

\begin{tabular}{|c|c|c|c|c|}
\hline Authors & Approach & Task \& Stimuli & $\begin{array}{l}\text { SRPE/ } \\
\text { URPE }\end{array}$ & $\begin{array}{l}\text { Effect } \\
\text { on } \\
\text { memory }\end{array}$ \\
\hline Bunzeck et al. (2010) & $\begin{array}{l}\text { Reward } \\
\text { prediction }\end{array}$ & $\begin{array}{l}\text { Each of three cues (colored squares) is } \\
\text { followed by one of two potential reward } \\
\text { values (medium-low, medium-high, and low- } \\
\text { high), so a medium reward can be better or } \\
\text { worse than expected. After reward feedback, a } \\
\text { novel (indoor or outdoor) scene is presented. } \\
\text { Scene recognition is probed after a one-day } \\
\text { delay. }\end{array}$ & SRPE & Positive \\
\hline $\begin{array}{l}\text { De Loof et al. (2018) } \\
\text { (see also Figure 1a-b) }\end{array}$ & $\begin{array}{l}\text { Reward } \\
\text { prediction }\end{array}$ & $\begin{array}{l}\text { On each trial, participants see one Dutch word } \\
\text { together with four (trial-novel) Swahili words } \\
\text { and choose a translation from either one, two } \\
\text { or four of these Swahili words. Manipulating } \\
\text { the number of eligible options }(1,2 \text {, or } 4 \text { ) and } \\
\text { whether a trial is rewarded or not, allowed } \\
\text { manipulation of RPEs. For example, in the } \\
\text { case of a four-option, rewarded trial, } \\
\text { participants experience a RPE of } 1-1 / 4=.75 \text {; } \\
\text { in case of a two-option, non-rewarded trial, } \\
\text { participants exhibit a RPE of } 0-1 / 2=-.50 \text {. }\end{array}$ & SRPE & Positive \\
\hline Davidow et al. (2016) & $\begin{array}{l}\text { Reward } \\
\text { prediction }\end{array}$ & $\begin{array}{l}\text { A cue is presented with two targets linked to } \\
\text { different reward values. Subjects must (learn } \\
\text { to predict and) choose the high-value target. } \\
\text { Trial-novel images are shown during } \\
\text { subsequent reward feedback. Image memory } \\
\text { is probed afterwards via old/new judgements. }\end{array}$ & SRPE & Positive \\
\hline $\begin{array}{l}\text { Rouhani et al. (2018) } \\
\text { (see also Figure 1c-d) }\end{array}$ & $\begin{array}{l}\text { Reward } \\
\text { prediction }\end{array}$ & $\begin{array}{l}\text { Participants track the reward associated with } \\
\text { different indoor and outdoor scenes. On each } \\
\text { trial, participants predict the reward (for a } \\
\text { particular scene), and subsequently receive } \\
\text { feedback about their estimate. From this } \\
\text { difference (feedback - predicted reward), a }\end{array}$ & URPE & Positive \\
\hline
\end{tabular}


Jang et al. (2019)

(see also Figure 1e-f)

Wimmer et al. (2014)

Butterfield \& Metcalfe (2001)

Metcalfe et al. (2012)

Pine et al. (2018)
RPE can be calculated. Scene memory is probed after this initial task via old/new judgments.

Reward prediction

On each trial, participants see a value and a stimulus (animate or inanimate) for that trial, and decide to play or pass on that trial (Figure 1e). After each choice, the image is shown with reward feedback. Afterwards, recognition memory for the images is probed via old/new judgements.

Reward prediction

Participants track the drifting reward probability of colored squares, which are overlaid with incidental trial-unique images and followed by feedback. Recognition memory for the images is probed via old/new judgements after a one-day delay.

Multiple Participants are presented with questions for repetition which they have to generate an answer and rate their confidence, followed by a surprise retest.

Multiple

repetition

Participants are presented with general information questions. In a first test phase, participants provide answers and rate their confidence. In the subsequent phase, subjects received feedback about their answers.

Finally, participants are retested on a subset of questions in a second test phase.

Multiple Participants study a text and are tested after repetition two days, at which time they also provide confidence ratings for their answers. On a small fraction of trials, participants receive false feedback (i.e., trials that were answered correctly but labeled as false), and received novel feedback (i.e., a novel "correct" answer)
SRPE Positive

SRPE Negative

URPE Positive

URPE Positive

URPE Positive 
on those trials. A second (incidental) test is given after 7 days. 


\section{References}

3671 Squire, L.R. (2004) Memory systems of the brain: A brief history and current

$368 \quad$ perspective. Neurobiol. Learn. Mem. 82, 171-177

3692 Wang, J.X. et al. (2018) Prefrontal cortex as a meta-reinforcement learning system.

370 Nat. Neurosci. 21, 860-868

3713 Sutton, R.S. and Barto, A.G. (2018) Reinforcement Learning : An Introduction, MIT $372 \quad$ Press.

3734 Rao, R.P. and Ballard, D.H. (1999) Predictive coding in the visual cortex: a functional interpretation of some extra-classical receptive-field effects. Nat. Neurosci. 2, 79-87

3755 Friston, K.J. (2003) Learning and inference in the brain. Neural Netw. 16, 1325-52

3766 Den Ouden, H.E.M. et al. (2012) How prediction errors shape perception, attention, $377 \quad$ and motivation. Front. Psychol. 3, 1-12

3787 Rescorla, R.A. and Wagner, A.R. (1972) A theory of Pavlovian conditioning:

379 Variations in the effectiveness of reinforcement and nonreinforcement. In Classical 380 conditioning II: Current research and theory (Blake, A. H. and Prokasy, W. F., eds), 381 pp. 64-99, Appleton-Century-Croft, New York

3828 Ljungberg, T. et al. (1992) Responses of monkey dopamine neurons during learning of 383 behavioral reactions. J. Neurophysiol. 67, 145-63

3849 Eshel, N. et al. (2016) Dopamine neurons share common response function for reward $385 \quad$ prediction error. Nat. Neurosci. 19, 479-86

38610 Mnih, V. et al. (2015) Human-level control through deep reinforcement learning.

$387 \quad$ Nature $518,529-533$

38811 Silver, D. et al. (2016) Mastering the game of Go with deep neural networks and tree $389 \quad$ search. Nature 529, 484-489 
39012 Pearce, J.M. and Hall, G. (1980) A model for Pavlovian learning: Variations in the

391 effectiveness of conditioned but not of unconditioned stimuli. Psychol. Rev. 87, 532-

$392 \quad 552$

39313 Dayan, P. et al. (2000) Learning and selective attention. Nat. Neurosci. 3 Suppl, 1218$394 \quad 23$

39514 Behrens, T.E.J. et al. (2007) Learning the value of information in an uncertain world. Nat. Neurosci. 10, 1214-21

39715 Silvetti, M. et al. (2018) Dorsal anterior cingulate-brainstem ensemble as a reinforcement meta-learner. PLOS Comput. Biol. DOI:

$399 \quad$ https://doi.org/10.1371/journal.pcbi.1006370

40016 Courville, A.C. et al. (2006) Bayesian theories of conditioning in a changing world. Trends Cogn. Sci. 10, 294-300

40217 Roesch, M.R. et al. (2012) Surprise ! Neural correlates of Pearce - Hall and Rescorla Wagner coexist within the brain. Eur. J. Neurosci. 35, 1190-1200

40418 Bunzeck, N. et al. (2010) A Common Mechanism for Adaptive Scaling of Reward and $405 \quad$ Novelty. Hum. Brain Mapp. 1394, 1380-1394

40619 Mason, A. et al. (2017) Adaptive scaling of reward in episodic memory : a replication $407 \quad$ study. Q. J. Exp. Psychol. 70, 2306-2318

40820 Mason, A. et al. (2017) The role of reward and reward uncertainty in episodic 409 memory. J. Mem. Lang. 96, 62-77

41021 De Loof, E. et al. (2018) Signed reward prediction errors drive declarative learning. PLoS One 13(1), e0189212

41222 Buc Calderon, C. et al. (2020) Signed reward prediction errors in the ventral striatum $413 \quad$ drive episodic memory. bioarxiv

41423 Davidow, J.Y. et al. (2016) An Upside to Reward Sensitivity: The Hippocampus 
Supports Enhanced Reinforcement Learning in Adolescence. Neuron 92, 93-99

24 Wimmer, G.E. et al. (2014) Episodic Memory Encoding Interferes with Reward Learning and Decreases Striatal Prediction Errors. J. Neurosci. 34, 14901-14912

25 Rouhani, N. et al. (2018) Dissociable Effects of Surprising Rewards on Learning and Memory. J. Exp. Psychol. Learn. Mem. Cogn. 44, 1430-1443

26 Jang, A.I. et al. (2019) Positive reward prediction errors during decision-making strengthen memory encoding. Nat. Hum. Behav. DOI: 10.1038/s41562-019-0597-3

27 Aberg, K.C. et al. (2017) Trial-by-Trial Modulation of Associative Memory Formation by Reward Prediction Error and Reward Anticipation as Revealed by a Biologically Plausible Computational Model. Front. Hum. Neurosci. 11, 56

28 Satterthwaite, T.D. et al. (2012) Being right is its own reward: load and performance related ventral striatum activation to correct responses during a working memory task in youth. Neuroimage 61, 723-9

29 Metcalfe, J. (2017) Learning from errors. Annu. Rev. Psychol. 68, 465-489

30 Metcalfe, J. and Finn, B. (2011) People's hypercorrection of high-confidence errors: Did they know it all along? J. Exp. Psychol. Learn. Mem. Cogn. 37, 437-448

31 Butterfield, B. and Metcalfe, J. (2006) The correction of errors committed with high confidence. Metacognition Learn. DOI: 10.1007/s11409-006-6894-z

32 Fazio, L.K. and Marsh, E.J. (2009) Surprising feedback improves later memory. Psychon. Bull. Rev. 16, 88-92

33 Butterfield, B. and Metcalfe, J. (2001) Errors Committed With High Confidence Are Hypercorrected. 27, 1491-1494

34 Pine, A. et al. (2018) Knowledge acquisition is governed by striatal prediction errors. Nat. Commun. 9, 1673

35 Ergo, K. et al. (2019) Oscillatory signatures of reward prediction errors in declarative 
$440 \quad$ learning. Neuroimage $186,137-145$

44136 Cavanagh, J.F. and Frank, M.J. (2014) Frontal theta as a mechanism for cognitive

$442 \quad$ control. Trends Cogn. Sci. 18, 414-421

$443 \quad 37$ Kleberg, F.I. et al. (2014) Ongoing theta oscillations predict encoding of subjective

444 memory type. Neurosci. Res. 83, 69-80

44538 HajiHosseini, A. et al. (2012) The role of beta-gamma oscillations in unexpected

$446 \quad$ rewards processing. Neuroimage $60,1678-1685$

44739 Arnold, K.M. and Mcdermott, K.B. (2013) Test-Potentiated Learning : Distinguishing 448 Between Direct and Indirect Effects of Tests. J. Exp. Psychol. Learn. Mem. Cogn. 39, $449 \quad 940-945$

45040 Pastötter, B. and Bäuml, K.T. (2014) Retrieval practice enhances new learning: the $451 \quad$ forward effect of testing. Front. Psychol. 5, 1-5

45241 Braun, E.K. et al. (2019) Retroactive and graded prioritization of memory by reward. 453 Nat. Commun. DOI: 10.1038/s41467-018-07280-0

45442 Fries, P. (2015) Rhythms for Cognition: Communication through Coherence. Neuron $455 \quad 88,220-235$

45643 Backus, A.R. et al. (2016) Hippocampal-Prefrontal Theta Oscillations Support 457 Memory Integration. Curr. Biol. 26, 1-8

45844 Wang, D. et al. (2018) Single-trial Phase Entrainment of Theta Oscillations in Sensory 459 Regions Predicts Human Associative Memory Performance. J. Neurosci. 38, 6299$460 \quad 6309$

46145 Clouter, A. et al. (2017) Theta Phase Synchronization Is the Glue that Binds Human 462 Associative Memory. Curr. Biol. 27, 3143-3148

46346 Kaminski, J. et al. (2018) Novelty-Sensitive Dopaminergic Neurons in the Human 464 Substantia Nigra Predict Success of Declarative Memory Formation. Curr. Biol. 28, 
46647 Berens, S.C. and Horner, A.J. (2017) Theta rhythm: Temporal glue for episodic memory. Curr. Biol. 27, R1110-R1112

48 Verbeke, P. and Verguts, T. (2019) Learning to synchronize: How biological agents can couple neural task modules for dealing with the stability-plasticity dilemma. PLoS Comput. Biol.

47149 Lisman, J.E. et al. (2011) A neoHebbian framework for episodic memory; role of dopamine-dependent late LTP. Trends Neurosci. 34, 536-47

47350 Matsumoto, M. and Hikosaka, O. (2009) Two types of dopamine neuron distinctly $474 \quad$ convey positive and negative motivational signals. Nature 459, 837-41

47551 Wagatsuma, A. et al. (2017) Locus coeruleus input to hippocampal CA3 drives single$476 \quad$ trial learning of a novel context. Proc. Natl. Acad. Sci. DOI: 10.1073/pnas. 1714082115

47852 Lisman, J.E. and Grace, A. a (2005) The hippocampal-VTA loop: controlling the entry 479 of information into long-term memory. Neuron 46, 703-13

48053 Takahashi, Y.K. et al. (2016) Temporal Specificity of Reward Prediction Errors Signaled by Putative Dopamine Neurons in Rat VTA Depends on Ventral Striatum. Neuron 91, 182-193

48354 Wittmann, B.C. et al. (2005) Reward-related FMRI activation of dopaminergic 484 midbrain is associated with enhanced hippocampus-dependent long-term memory $485 \quad$ formation. Neuron $45,459-67$

48655 Gruber, M.J. et al. (2016) Post-learning hippocampal dynamics promote preferential 487 retention of rewarding events. Neuron $89,1110-1120$

48856 Patil, A. et al. (2016) Reward retroactively enhances memory consolidation for related 489 items. Learn. Mem. 24, 65-69 
49057 Miendlarzewska, E.A. et al. (2016) Influence of reward motivation on human

491 declarative memory. Neurosci. Biobehav. Rev. 61, 156-176

49258 Alberini, C.M. and Ledoux, J.E. (2013) Memory reconsolidation. Curr. Biol. 23,

$493 \quad$ R746-R750

49459 Fernández, R.S. et al. (2016) The fate of memory : Reconsolidation and the case of

495 Prediction Error. Neurosci. Biobehav. Rev. 68, 423-441

49660 Nader, K. et al. (2000) Fear memories require protein synthesis in the amygdala for

497 reconsolidation after retrieval. Nature 406, 722-726

49861 Sinclair, A.H. and Barense, M.D. (2018) Surprise and destabilize : prediction error

499 influences episodic memory reconsolidation. Learn. Mem. 25, 369-381

50062 Exton-McGuinness, M.T.J. et al. (2015) Updating memories - The role of prediction

501 errors in memory reconsolidation. Behav. Brain Res. 278, 375-384

50263 Sevenster, D. et al. (2013) Prediction Error Governs Pharmacologically Induced

$503 \quad$ Amnesia for Learned Fear. Science (80-. ). 339, 830-833

50464 Sinclair, A.H. and Barense, M.D. (2019) Prediction Error and Memory Reactivation :

$505 \quad$ How Incomplete Reminders Drive Reconsolidation. Trends Neurosci. DOI:

$506 \quad$ 10.1016/j.tins.2019.08.007

50765 Eichenbaum, H. (2004) Hippocampus: Cognitive processes and neural representations

508 that underlie declarative memory. Neuron 44, 109-120

50966 Metcalfe, J. et al. (2012) Neural Correlates of People's Hypercorrection of Their False

$510 \quad$ Beliefs. J. Cogn. Neurosci. 24, 1571-1583

511

512 
Reward prediction error and declarative memory

\section{Acknowledgements}

514 KE and TV were supported by project G012816N from the Research Council Flanders

515 (FWO). KE is a research fellow at the Research Council Flanders. EDL and TV were

516 supported by project BOF17-GOA-004 awarded by the Ghent University Research Council. 